\title{
EX-PRESS Glaucoma Filtration Device: efficacy, safety, and predictability
}

This article was published in the following Dove Press journal:

Medical Devices: Evidence and Research

2 September 2015

Number of times this article has been viewed

\section{Jessica E Chan \\ Peter A Netland}

Department of Ophthalmology, University of Virginia School of Medicine, Charlottesville, VA, USA

Correspondence: Peter A Netland Department of Ophthalmology, University of Virginia School of Medicine, PO Box 8007I5, Charlottesville, VA 22908-07I5, USA

Tel + I 4349821086

Fax + I 4349245180

Email pnetland@virginia.edu

\begin{abstract}
Trabeculectomy has been the traditional primary surgical therapy for open-angle glaucoma. While trabeculectomy is effective in lowering intraocular pressure, complications associated with the procedure have motivated the development of alternative techniques and devices, including the EX-PRESS Glaucoma Filtration Device. This review describes the efficacy, safety, complication rates, and potential advantages and disadvantages of the EX-PRESS Glaucoma Filtration Device. EX-PRESS implantation is technically simpler compared with that of trabeculectomy, with fewer surgical steps. Vision recovery has been more rapid after EX-PRESS implantation compared with trabeculectomy. Intraocular pressure variation is lower during the early postoperative period, indicating a more predictable procedure. While efficacy of the EX-PRESS implant has been comparable to trabeculectomy, postoperative complications appear less common after EX-PRESS implantation compared with trabeculectomy. The EX-PRESS Glaucoma Filtration Device appears to be safe and effective in the surgical management of open-angle glaucoma.
\end{abstract}

Keywords: surgery, trabeculectomy, mitomycin C, glaucoma drainage implant

\section{Introduction}

Glaucoma is the leading cause of irreversible blindness worldwide, with an estimated 64.3 million people affected globally in $2013 .{ }^{1}$ These numbers are projected to further increase to 76 million glaucoma cases worldwide in 2020, and 111.8 million in $2040 .{ }^{1}$ The goal of treatment in glaucoma is to lower the intraocular pressure. Therapies include medical, laser, and surgical interventions, with surgery typically reserved for cases of progressive disease or risk of progression due to uncontrolled intraocular pressure.

Trabeculectomy has been considered the conventional primary surgical procedure for treatment of glaucoma. Over time, the procedure has been modified to include use of adjunctive antifibrosis drugs that improve efficacy and lower mean postoperative intraocular pressure. Although highly effective in lowering the intraocular pressure, trabeculectomy is associated with poor predictability and numerous minor, as well as potentially vision-threatening, complications, including hypotony, choroidal detachment, suprachoroidal hemorrhage, and vision loss. Complications have persisted and visual recovery remains slow, despite modification of the procedure to include laser suture lysis or releasable sutures during the immediate postoperative period. Various surgical devices and techniques have been developed, with the goal of attaining low intraocular pressure while minimizing the risk of complications to the patient.

The EX-PRESS Glaucoma Filtration Device (Alcon Laboratories, Fort Worth, TX, USA) was approved in 2002 by the United States Food and Drug Administration. It is a stainless steel, nonvalved filtration device designed to shunt aqueous humor 
from the anterior chamber to a subconjunctival filtration bleb (Figure 1). Biocompatibility of the device was first demonstrated in rabbit eyes, with little to no inflammation found on histopathologic examination. ${ }^{2}$ The device is $2.64 \mathrm{~mm}$ in length, and is available with either a 50 or $200 \mu \mathrm{m}$ internal lumen size. Originally designed for placement directly underneath the conjunctiva, the procedure initially was associated with complications such as conjunctival erosion, extrusion, hypotony, and other adverse effects. ${ }^{3-5}$ The procedure was modified by placing the device underneath a partial-thickness scleral flap, largely eliminating the risk of hypotony, erosion, and extrusion. ${ }^{6}$ Placement of the EX-PRESS device under a partial-thickness scleral flap has been widely adopted and is now the recommended technique for device placement.

There is a growing body of literature assessing the EX-PRESS Glaucoma Filtration Device and comparing its safety and efficacy to trabeculectomy. This review aims at summarizing the evidence to date on the outcomes, safety, and acceptability for usage of this device in patients with glaucoma.

\section{Indications for usage}

The EX-PRESS Glaucoma Filtration Device has been approved for usage for lowering intraocular pressure in patients who have uncontrolled glaucoma, including those who have failed prior medical and conventional surgical treatments. As the device is placed in the anterior chamber angle, its usage is contraindicated in patients with acuteangle-closure glaucoma. Caution is advised in patients with narrow angles unless the procedure will be combined with cataract surgery. However, the risk of occlusion of the device is low using the current version of the device, which is shorter than the original version. Patients with well-controlled uveitis may be treated, although patients with active uveitis may develop occlusion of the device. Cautious usage of the device is recommended in young patients, due to the limited information about long-term follow-up with the implant. ${ }^{7}$

\section{Efficacy in noncomparative trials}

This review will focus on the results of the standard method for surgical implantation of the EX-PRESS implant, which includes placement of the device underneath a partial-thickness scleral flap. The results of noncomparative studies have indicated that the EX-PRESS device has acceptable rates of success and intraocular pressure lowering in both the short and long terms.

The initial study of EX-PRESS implantation underneath a scleral flap was a noncomparative study of 24 eyes of open-angle glaucoma patients who had failed medical treatment and prior surgical treatment. ${ }^{6}$ In this study, the intraocular pressure was reduced from a mean of $27.2 \pm 7.1 \mathrm{mmHg}$ preoperatively to $14.5 \pm 5.0 \mathrm{mmHg}$ at 12 months $(\mathrm{n}=21)$, and $14.2 \pm 4.2 \mathrm{mmHg}$ at 24 months $(\mathrm{n}=8)$ postoperatively, with $P<0.05$ across all time points. More recently, Lankaranian et $\mathrm{al}^{8}$ performed a noncomparative study on outcomes of the EX-PRESS implant in 100 patients with glaucoma and a history of either previous cataract or failed glaucoma surgeries. Success was defined as complete if intraocular pressure was 5-21 mmHg without medication or surgical intervention, and qualified if intraocular pressure was within the same range, but with glaucoma medication. Complete success was found in $60 \%$ of patients and $24 \%$ were qualified successes, with a decline in intraocular pressure from a mean preoperative intraocular pressure of $27.7 \pm 9.2 \mathrm{mmHg}$ to $14.02 \pm 5.1 \mathrm{mmHg}$ at last follow-up.

Long-term outcomes after implantation of the EX-PRESS device were reported in a retrospective review of the records of 248 patients who were treated with the EX-PRESS implant. ${ }^{9}$ One hundred and thirty-six eyes (55\%) underwent EX-PRESS implantation alone, while 112 eyes (45\%) had combined
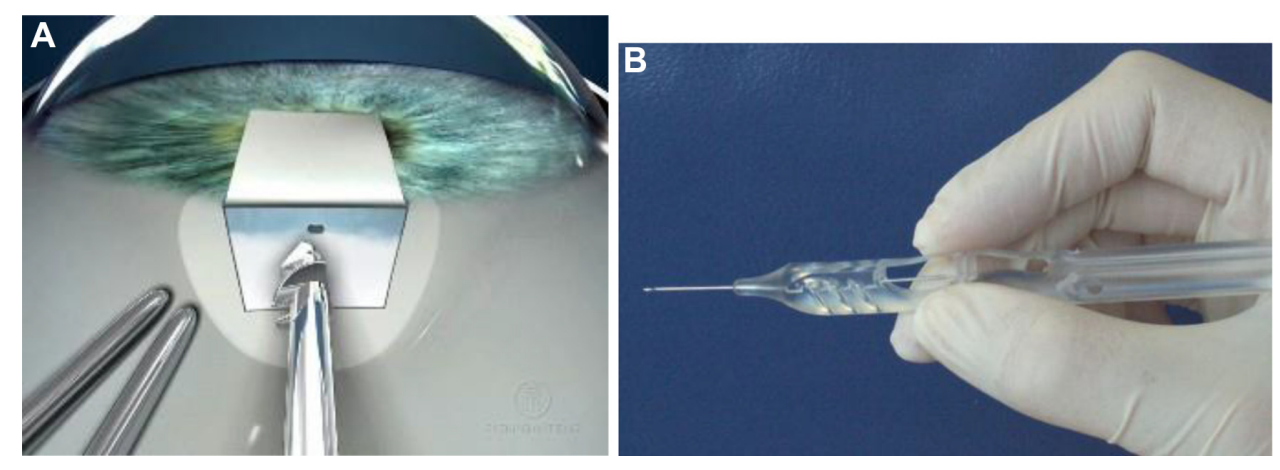

Figure I The EX-PRESS Glaucoma Filtration Device is placed under a partial-thickness scleral flap through a 25-gauge needle tract at the limbus.

Notes: (A) The Model P-50 device has an inner diameter of $50 \mu \mathrm{m}$, an outer diameter of $0.4 \mathrm{~mm}$, and is $2.64 \mathrm{~mm}$ long. (B) The EX-PRESS device is preloaded for implantation on a handheld delivery system. Images provided courtesy of Alcon, Inc. 
cataract extraction with EX-PRESS implantation, with the results of both groups pooled together. The mean preoperative intraocular pressure decreased from $27.63 \pm 8.26 \mathrm{mmHg}$ $(n=248)$ to $13.95 \pm 2.70 \mathrm{mmHg}(n=95)$ at 5 years. With a definition of complete success as a postoperative intraocular pressure of 5-18 $\mathrm{mmHg}$ without glaucoma medications, and a qualified success as the same intraocular pressure range but with or without medications, the complete and qualified success rates were $83 \%$ and $85 \%$, respectively, at 1 year, and $57 \%$ and $63 \%$, respectively, at 5 years follow-up.

\section{Comparison of EX-PRESS with trabeculectomy}

Because trabeculectomy is the conventional standard for primary glaucoma surgery, studies have compared the results of EX-PRESS device implantation to that of trabeculectomy (Figure 2). The initial comparison of results after EX-PRESS implantation to trabeculectomy was a retrospective series by Maris et al, ${ }^{10}$ comparing 50 eyes that underwent EX-PRESS implantation with 50 matched control eyes treated with trabeculectomy. Both groups included patients who also had concomitant phacoemulsification at the time of glaucoma surgery. Success was defined as intraocular pressure between 5 and $21 \mathrm{mmHg}$, with or without glaucoma medications, and without further glaucoma surgery or removal of the implant. In the early postoperative period, the trabeculectomy group had a statistically significant lower mean intraocular pressure than the EX-PRESS group; however, after 3 months, the reduction in intraocular pressure was similar in both groups, with a range of $39.9 \%-46.6 \%$ reduction in intraocular pressure in the EX-PRESS group, and a $28.6 \%-45.4 \%$ reduction

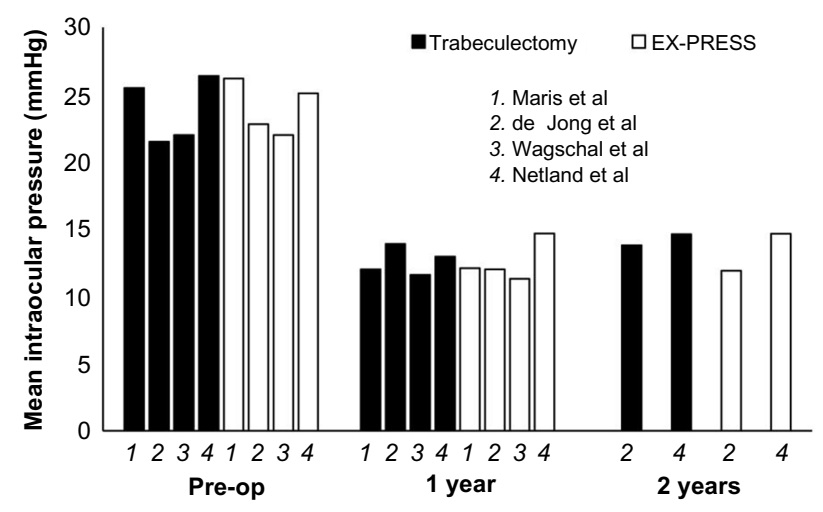

Figure 2 Mean intraocular pressure after EX-PRESS implantation and trabeculectomy. Notes: Studies showing specific intraocular pressure values at I and 2 years after surgery are shown. EX-PRESS device implantation achieves similar mean intraocular pressure compared with trabeculectomy, indicating that the procedure can achieve low target intraocular pressure in patients with advanced glaucoma damage. Data from Maris et al, ${ }^{10}$ de Jong et al, ${ }^{12}$ Netland et al, ${ }^{16}$ and Wagschal et al. ${ }^{17}$

Abbreviation: Pre-op; preoperative. in the trabeculectomy group. There was no significant difference in success rates between groups, with a $90.0 \%$ and $92.0 \%$ success rate at last follow-up in the EX-PRESS and trabeculectomy groups, respectively.

A prospective comparative study by de Jong ${ }^{11}$ examined the results of the EX-PRESS in 40 eyes in comparison to trabeculectomy in 40 eyes. Patients in the EX-PRESS group had a $42.0 \%$ reduction in intraocular pressure at 12 months, while patients in the trabeculectomy group had a $29.3 \%$ reduction $(P=0.05)$. Complete success was defined as intraocular pressure $4-18 \mathrm{mmHg}$ without the use of antiglaucoma medications, which was achieved in $81.8 \%$ of EX-PRESS patients and $47.5 \%$ of trabeculectomy patients $(P=0.002)$. The outcomes of these patients after a longer period of follow-up were then published in a subsequent paper by de Jong et al. ${ }^{12}$ Thirty-nine eyes in each group from the original study were included. The trend toward better intraocular pressure reduction in the EX-PRESS group versus the trabeculectomy group continued until year 3 . After year 3 and until year 5, the intraocular pressure difference between the two groups was not significant. Similarly, more patients achieved a complete success in the EX-PRESS group than the trabeculectomy group at 3 years $(66.7 \%$ versus $41.0 \%, P=0.02$ ), but this difference was no longer statistically significant at 5 years $(59.0 \%$ versus $46.2 \%, P=0.25)$. Overall, these studies suggested that the intraocular pressure control with the EX-PRESS implant is comparable to that of trabeculectomy.

A retrospective series compared 35 consecutive EXPRESS procedures with 35 consecutive standard trabeculectomy procedures. ${ }^{13}$ The intraocular pressure in the EX-PRESS group was higher than that in the trabeculectomy group at 1 year and at last follow-up $(P=0.004$ and $P=0.008$, respectively). However, the overall reductions in intraocular pressure at last follow-up were similar, with a $45 \%$ reduction in the EX-PRESS group, and $48.45 \%$ in the trabeculectomy group $(P=0.209)$. In addition, success rates were not significantly different between the groups. Unqualified success was defined as an intraocular pressure of $5-18 \mathrm{mmHg}$ and at least a $30 \%$ reduction in intraocular pressure without the use of glaucoma medications and a qualified success as the same but with medications. Unqualified success was achieved in $77.14 \%$ of EX-PRESS procedures and $74.29 \%$ of trabeculectomies at last follow-up $(P=1.00)$, and qualified successes were found in an additional $5.71 \%$ of EX-PRESS eyes and $8.57 \%$ of trabeculectomy eyes $(P=0.99)$.

Additional studies have found comparable rates of success between eyes treated with EX-PRESS versus standard 
trabeculectomy. Marzette and Herndon ${ }^{14}$ retrospectively compared 77 eyes treated with trabeculectomy to 76 eyes treated with EX-PRESS implants, and included patients who also underwent phacoemulsification at the time of surgery. Complete success was defined as intraocular pressure of 5-21 $\mathrm{mmHg}$ without additional medication or glaucoma surgery, and qualified success as the same but with glaucoma medication. The overall success rate (total and qualified) was not statistically significantly different between the two groups, with $82 \%$ and $71 \%$ success rates in the EX-PRESS and trabeculectomy groups, respectively. There was no statistically significant difference in the percent reduction of intraocular pressure, with an average of $42 \%$ reduction in both groups. Moisseiev et $\mathrm{al}^{15}$ also performed a retrospective review comparing EX-PRESS in 39 eyes versus trabeculectomy in 61 eyes. In this study, there was no significant difference in intraocular pressure reduction or success rates between the two groups. Success was defined as a $20 \%$ reduction in intraocular pressure from the preoperative value or an intraocular pressure under $20 \mathrm{mmHg}$, with $86.9 \%$ of the trabeculectomy group, and $84.6 \%$ of the EX-PRESS group achieving surgical success.

The strongest evidence for the efficacy of the EX-PRESS device is reported in randomized prospective clinical trials. A large randomized prospective multicenter trial by Netland et $\mathrm{al}^{16}$ included 59 eyes treated with the EXPRESS implant and 61 eyes treated with trabeculectomy. The mean intraocular pressure was similar in both groups during postoperative follow-up, with a mean intraocular pressure of $14.7 \pm 4.6 \mathrm{mmHg}$ in the EX-PRESS group and $14.6 \pm 7.1 \mathrm{mmHg}$ in the trabeculectomy group at 2 years after surgery $(P=0.927)$. With success defined as intraocular pressure 5-18 mmHg with or without medications, and without further glaucoma surgery, success rates were 90\% and $87 \%$ at 1 year, and $83 \%$ and $79 \%$ at 2 years in the EX-PRESS and trabeculectomy groups, respectively $(P=0.563)$.

A prospective randomized study by Wagschal et $\mathrm{al}^{17}$ included 33 subjects receiving the EX-PRESS device and 31 underwent trabeculectomy. At 1 year, there was a similar reduction in intraocular pressure between groups, with a $47 \%$ reduction in the trabeculectomy group, and a $50 \%$ reduction in the EX-PRESS group. Mean intraocular pressure was not significantly different between groups at all visits. Complete success, defined as intraocular pressure between 5 and $18 \mathrm{mmHg}$ and a $20 \%$ reduction in intraocular pressure from baseline without medication, was found in 57\% of the trabeculectomy group and $70 \%$ of the EX-PRESS group $(P=0.28)$, while a qualified success (same definition but with hypotensive medications) was achieved by $77 \%$ in both groups.

In a prospective randomized study of 15 patients, Dahan et $\mathrm{al}^{18}$ compared trabeculectomy and EX-PRESS implantation in fellow eyes of the same patient. In this study, eyes treated with EX-PRESS implantation had significantly lower postoperative mean intraocular pressure and number of medications compared with the trabeculectomy group. Complete success rates (intraocular pressure 5-18 $\mathrm{mmHg}$ without medications) were higher after EX-PRESS implantation compared with trabeculectomy $(P=0.0024)$.

The results from randomized prospective clinical trials provide strong evidence that EX-PRESS implantation results in mean postoperative intraocular pressure reduction and surgical success rates are comparable to trabeculectomy. Clinicians recognize that trabeculectomy, although associated with complications, is an effective procedure in patients with advanced glaucoma damage who require postoperative intraocular pressure in the mid- to low teens. Thus, in our view, the EX-PRESS Glaucoma Filtration Device procedure is suitable for patients when indicated, including those patients with advanced glaucomatous damage to the optic nerve and visual fields.

\section{Visual recovery}

Several studies, including randomized clinical trials, have shown that surgery with the EX-PRESS implant is associated with more rapid postoperative visual recovery as compared to that with trabeculectomy. ${ }^{13,16,17}$ Good and Kahook ${ }^{13}$ observed visual recovery to near-baseline level of vision at 1 week postoperation after EX-PRESS implantation, as compared to 1 month after trabeculectomy. Wagschal et al ${ }^{17}$ found that visual acuity in patients treated with the EX-PRESS implant did not differ significantly from baseline by 1 month postoperation $(P=0.17)$, and remained stable for all subsequent visits. However, in the trabeculectomy group, the visual acuity remained significantly lower than baseline from 1 day to 1 year postoperation $(P<0.001)$.

In a randomized prospective trial, Netland et $\mathrm{al}^{16}$ found a significant reduction from baseline $(P<0.001)$ in vision at day 1 postoperatively in both groups, but a recovery of vision by 1 month in the EX-PRESS group $(P=0.285)$ and 3 months in the trabeculectomy group $(P=0.255)$, with no significant differences at any further time points. In another prospective randomized trial by Beltran-Agullo et al, ${ }^{19} 33$ patients randomized to EX-PRESS and 31 to trabeculectomy were analyzed and rates of visual recovery were compared. By month 1, visual acuity in the EX-PRESS group was no 
longer significantly different from baseline $(P=0.23)$ and remained stable throughout 6 months of follow-up. In contrast, in the trabeculectomy group, the visual acuity remained significantly lower than baseline throughout all 6 months of follow-up. Furthermore, 47\% of trabeculectomy eyes versus $16 \%$ of EX-PRESS eyes lost $\geq 2$ Snellen lines $(P=0.01)$ at 6 months.

\section{EX-PRESS implantation combined with cataract surgery}

Evidence thus far seems to indicate that combining cataract surgery with EX-PRESS implantation leads to favorable outcomes. ${ }^{10,14,15}$ Efficacy analysis of intraocular pressure and surgical success comparing patients treated with or without concurrent phacoemulsification at the time of EX-PRESS surgery failed to show a significant difference in either treatment group at last follow-up in studies by Maris et al, ${ }^{10}$ Marzette and Herndon, ${ }^{14}$ and Moisseiev et al. ${ }^{15}$

Kanner et $\mathrm{al}^{20}$ performed a comparative consecutive case series analyzing the results of 231 eyes receiving the EX-PRESS alone versus 114 eyes that underwent combined phacoemulsification with EX-PRESS implantation. In both groups, there was a significant decrease in intraocular pressure from baseline postoperatively. The change from baseline intraocular pressure was significantly greater after EX-PRESS implant alone compared with combined surgery $(P<0.001)$, which was similar to the differences observed in other studies of trabeculectomy alone compared with combined cataract surgery and trabeculectomy. Success was defined as intraocular pressure between 5 and $21 \mathrm{mmHg}$ with or without medications, but without further surgery or total loss of vision, with both groups achieving comparable rates of success at 3 years of $94.8 \%$ and $95.6 \%$ in the EX-PRESS and combined groups, respectively ( $P=0.948)$. Complication rates were low and were not statistically significant between groups.

\section{Safety and complications}

Reported complications from EX-PRESS implantation underneath a partial thickness scleral flap include hypotony, bleb leaks, and choroidal effusions, as expected with a procedure causing transscleral flow of aqueous. ${ }^{6,8-11,13-17}$ Although the rates of complications vary across studies, multiple studies have confirmed similar types of complications from the EX-PRESS as compared to that from trabeculectomy, ${ }^{10,11,14-17}$ with most of these studies powered to detect differences in intraocular pressure between groups. Several studies found fewer cases of hyphema ${ }^{13,16,17}$ and less hypotony after EX-PRESS implantation compared with trabeculectomy. ${ }^{10,13,14}$ Proposed explanations for the observation of decreased rates of hypotony during the early postoperative period following EX-PRESS implantation compared with trabeculectomy include flow restriction due to the small lumen $(50-200 \mu \mathrm{m})$ of the device (compared with the variable and large opening size after trabeculectomy), better seal of the scleral flap against the device compared with a sclerostomy, and greater predictability of the procedure with less variance of the early postoperative intraocular pressure. There have been rare reports of implant extrusion or conjunctival erosion since modification of the procedure with placement of the device under a partial thickness scleral flap.

Randomized prospective clinical trials have provided information about complications after implantation of the EX-PRESS compared with trabeculectomy. In the randomized multicenter prospective trial by Netland et al, ${ }^{16}$ the total number of postoperative complications was higher in the trabeculectomy group compared with the EX-PRESS group $(P=0.013)$. The number of subjects with at least one complication was ten $(17.0 \%)$ and $22(36.1 \%)$ in the EX-PRESS and trabeculectomy groups, respectively $(P=0.031)$. In the randomized prospective study by Wagschal et al, ${ }^{17}$ the study had a power of $80 \%$ to detect a $2.0 \mathrm{mmHg}$ intraocular pressure difference between groups, and was not designed to detect differences of complications. Nonetheless, hyphema was found more frequently in the trabeculectomy group when compared with the EX-PRESS group. In the randomized prospective study by Dahan et al, ${ }^{18}$ postoperative complications were more frequent after trabeculectomy (33\%) compared with after EX-PRESS (20\%). Significantly, more patients required at least one postoperative intervention for complications after trabeculectomy (27\%) versus those with EX-PRESS implantation $(0 \%, P=0.0009)$.

Useful information about low-frequency device-related complications has been reported in retrospective studies with large numbers of patients. In the retrospective comparative study by Kanner et al, ${ }^{20}$ the most common device-related complication in 345 patients was obstruction of the lumen of the device, which occurred in six patients $(1.7 \%)$ manifested by increased intraocular pressure and a low or flat bleb. In this study, all patients who developed this complication were successfully treated with Nd:YAG laser to the tip of the tube in the anterior chamber. A report by Bagnis et $\mathrm{al}^{21}$ confirmed the usefulness of the Nd:YAG laser as a therapeutic option to manage obstruction of the tube after implantation of the EX-PRESS device. Obstruction usually occurs inside the lumen of the device, where it may not be visualized by 
gonioscopy, at the point where the internal diameter constricts to $50 \mu \mathrm{m} .{ }^{22}$ This constriction point is sufficiently close to the opening into the anterior chamber to allow effective treatment with the Nd:YAG laser. In our patients, active uveitis may be associated with internal obstruction of the device. In rare instances, obstruction of the internal opening of the device with iris tissue or vitreous can occur, although the shorter shaft of the current implant (compared with previous models of the implant) and the openings on the side of the shaft of the device are intended to reduce the frequency of this problem.

Although most studies in the literature have not been designed to assess complications, evidence in the literature indicates fewer complications after EX-PRESS implantation compared with after trabeculectomy.

\section{Magnetic resonance imaging safety}

Although the EX-PRESS implant is composed of nonferomagnetic surgical stainless steel, the safety of magnetic resonance imaging (MRI) for these patients has arisen as a potential concern. Geffen et $\mathrm{al}^{23}$ studied the effect of a magnetic field of 1.5 and $3 \mathrm{~T}$ on an EX-PRESS implant in various conditions, including in human cadaver eyes, and concluded that MRI up to $3 \mathrm{~T}$ was likely safe in eyes with the EX-PRESS, due to ocular tissues preventing movement of the device. Seibold et $\mathrm{al}^{24}$ examined the movement of the EX-PRESS in various magnetic fields, and also found that the device was likely safe for MRI up to $3 \mathrm{~T}$. The effect of the device on imaging quality has been addressed in several studies. A small consecutive case series by De Feo et $\mathrm{al}^{25}$ found that the implant caused minimal artifacts on MRI of the orbit and brain, although the imaging quality of the optic nerve itself may have been slightly decreased. Mabray et $\mathrm{al}^{26}$ confirmed in their small retrospective study that the presence of an EX-PRESS in patient eyes did not cause any significant artifacts affecting the diagnostic interpretation of their MRI scans.

\section{Variance of intraocular pressure during the early postoperative period}

The surgical procedure for the implantation of the EX-PRESS device does not require a sclerostomy and peripheral iridectomy, unlike trabeculectomy. In contrast with trabeculectomy, there is no tissue excised during the EX-PRESS procedure, and less manipulation of ocular tissues may potentially reduce postoperative inflammation. These additional steps introduce some variability to the trabeculectomy procedure, because the sclerostomy and iridectomy will not be of uniform size from one surgery to the next. Placement of the EX-PRESS device through an opening created with a 25 -gauge needle is more straightforward and may be associated with more easily reproducible results. This may make the procedure simpler and more standardized, which may be beneficial for those surgeons who do not routinely perform glaucoma surgery.

In their prospective randomized trial, Netland et al ${ }^{16}$ observed less variance in the early postoperative intraocular pressure measurements after EX-PRESS implantation versus trabeculectomy. During the first 2 weeks after surgery, the intraocular pressure was recorded at the beginning of the visit, and then again after any treatments including laser suture lysis, removal of releasable sutures, or digital massage. The variance in both initial and final intraocular pressure measurements during the early postoperative period was significantly lower after EX-PRESS implantation as compared to that after trabeculectomy (Figure 3). It was hypothesized that this difference of variance could reflect the difference in surgical techniques between EX-PRESS surgery and trabeculectomy, with EX-PRESS being more standardized and thus less likely to lead to variability. Similar findings were subjectively observed but not statistically analyzed in a previous study. ${ }^{27}$ A retrospective study showed significantly fewer postoperative visits after EX-PRESS implant compared with those after trabeculectomy, ${ }^{13}$ presumably due to less

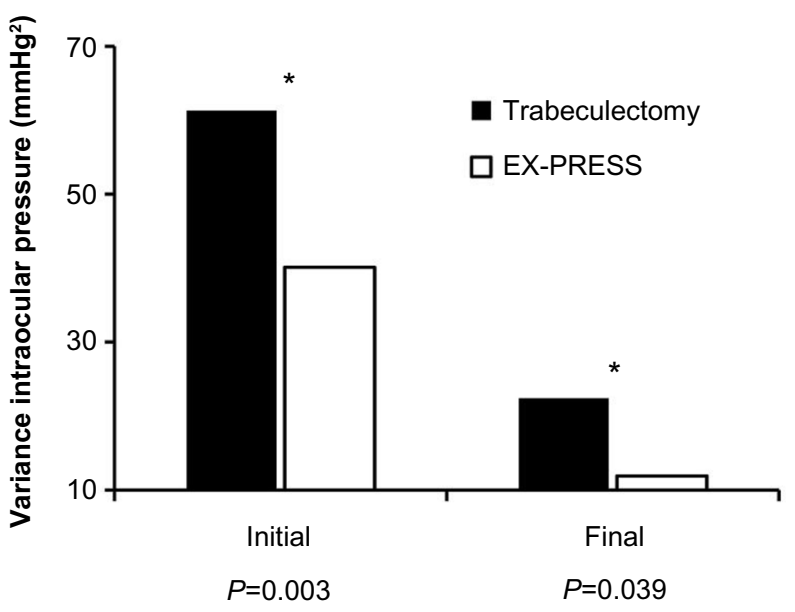

Figure 3 Variance of intraocular pressure during the early postoperative period after treatment with the EX-PRESS Glaucoma Filtration Device versus trabeculectomy. Notes: The variance of intraocular pressure during the early postoperative period was significantly lower after EX-PRESS implant under scleral flap compared with trabeculectomy. Initial values are before any treatment, and final values are after laser suture lysis, removal of releasable sutures, or digital massage. Values of variance are $\mathrm{mmHg}^{2}$ (square of $\mathrm{mmHg}$ ). $P$-values shown are for the Brown and Forsythe test of equal variance. (Asterisks $\left[{ }^{*}\right]$ indicate statistically significant difference). Lower variance of the intraocular pressure during the early postoperative period reflects greater predictability of the EX-PRESS procedure as compared with trabeculectomy. Data from Netland et al. ${ }^{16}$ 
variability and greater predictability of clinical results after EX-PRESS implant surgery.

Variance can be viewed as a statistical manifestation of surgical "predictability," with lower variance associated with a more "predictable" procedure. With greater variance associated with trabeculectomy, we anticipate increased high and low intraocular pressure during the early postoperative period. We also expect increased numbers of cases in the low (hypotony) range, because the variance is greater than the mean during the early postoperative period. Conversely, a lower variance associated with the EX-PRESS implant does not mean that hypotony cannot occur, but rather that it is less likely to occur.

\section{Cost considerations}

A consideration with clinical use of the EX-PRESS implant is the cost of the procedure. Patel et $\mathrm{a}^{28}$ compared the surgical and 1 year postoperative costs of EX-PRESS versus trabeculectomy in 43 subjects in Canada. They found no significant differences in the overall postoperative costs, costs of follow-up visits, additional procedures, and glaucoma medications between the two surgeries. However, the EX-PRESS had a net surgical cost that was greater than trabeculectomy, with the majority of the difference in that cost due to the cost of the device itself.

\section{Patient satisfaction and patient acceptability}

Clinicians have anecdotally described positive patient satisfaction and good patient acceptability of the EX-PRESS procedure. More rapid vision recovery would be expected to result in greater patient satisfaction with EX-PRESS device implantation compared with trabeculectomy. However, information to address this issue has not been reported in the literature. Thus, it is not possible to make evidence-based statements about the patient experience with the EX-PRESS procedure at this time.

\section{Conclusion}

Numerous retrospective comparative and prospective randomized studies, over a decade of clinical experience, have improved the understanding of the EX-PRESS Glaucoma Filtration Device. Clinicians can achieve similar intraocular pressure control and surgical success after both EX-PRESS implantation and trabeculectomy, which are procedures that have the potential to achieve low mean postoperative intraocular pressures and high long-term success rates. The EX-PRESS procedure can be effectively combined with phacoemulsification cataract surgery. The EX-PRESS procedure is more predictable than trabeculectomy procedure, with less variance of intraocular pressure during the early postoperative period. In addition, evidence in the literature indicates that recovery of vision is more rapid, and postoperative complications are less frequent after the EX-PRESS procedure when compared with trabeculectomy. The EX-PRESS Glaucoma Filtration Device has been a useful addition to the surgical treatment of glaucoma.

\section{Acknowledgment}

The prospective, multicenter, randomized trial of the EX-PRESS device performed by Dr Netland was an investigator-initiated trial, which received research support from Alcon Laboratories (Fort Worth, TX, USA). The sponsors had no role in the design or conduct of this research (clinical trials registration identifier NCT00444080; http:// www.clinicaltrials.gov).

\section{Disclosure}

The authors report no conflicts of interest in this work. Retrospective studies of the EX-PRESS device performed by Dr Netland received no sponsored support.

\section{References}

1. Tham YC, Li X, Wong TY, Quigley HA, Aung T, Cheng CY. Global prevalence of glaucoma and projections of glaucoma burden through 2040: a systematic review and meta-analysis. Ophthalmology. 2014;121: 2081-2090.

2. Nyska A, Glovinsky Y, Belkin M, Epstein Y. Biocompatibility of the Ex-PRESS miniature glaucoma drainage implant. J Glaucoma. 2003;12: 275-280.

3. Wamsley S, Moster MR, Rai S, Alvim HS, Fontanarosa J. Results of the use of the Ex-PRESS miniature glaucoma implant in technically challenging, advanced glaucoma cases: a clinical pilot study. Am J Ophthalmol. 2004;138:1049-1051.

4. Stewart RM, Diamond JG, Ashmore ED, Ayyala RS. Complications following Ex-Press glaucoma shunt implantation. Am J Ophthalmol. 2005;140:340-341.

5. Traverso CE, de Feo F, Messas-Kaplan A, et al. Long term effect on IOP of a stainless steel glaucoma drainage implant (Ex-PRESS) in combined surgery with phacoemulsification. Br J Ophthalmol. 2005;89: 425-429.

6. Dahan E, Carmichael TR. Implantation of a miniature glaucoma device under a scleral flap. J Glaucoma. 2005;14:98-102.

7. Salim S. The role of the Ex-PRESS glaucoma filtration device in glaucoma surgery. Semin Ophthalmol. 2013;28:180-184.

8. Lankaranian D, Razeghinejad MR, Prasad A, et al. Intermediateterm results of the Ex-PRESS miniature glaucoma implant under a scleral flap in previously operated eyes. Clin Experiment Ophthalmol. 2011;39:421-428.

9. Mariotti C, Dahan E, Nicolai M, Levitz L, Bouee S. Long-term outcomes and risk factors for failure with the EX-press glaucoma drainage device. Eye (Lond). 2014;28:1-8.

10. Maris PJ Jr, Ishida K, Netland PA. Comparison of trabeculectomy with Ex-PRESS miniature glaucoma device implanted under scleral flap. J Glaucoma. 2007;16:14-19. 
11. de Jong LA. The Ex-PRESS glaucoma shunt versus trabeculectomy in open-angle glaucoma: a prospective randomized study. Adv Ther. 2009;26:336-345.

12. de Jong L, Lafuma A, Aguadg AS, Berdeaux G. Five-year extension of a clinical trial comparing the EX-PRESS glaucoma filtration device and trabeculectomy in primary open-angle glaucoma. Clin Ophthalmol. 2011;5:527-533.

13. Good TJ, Kahook MY. Assessment of bleb morphologic features and postoperative outcomes after Ex-PRESS drainage device implantation versus trabeculectomy. Am J Ophthalmol. 2011;151:507-513.

14. Marzette L, Herndon LW. A comparison of the Ex-PRESSTM mini glaucoma shunt with standard trabeculectomy in the surgical treatment of glaucoma. Ophthalmic Surg Lasers Imaging. 2011;42:453-459.

15. Moisseiev E, Zunz E, Tzur R, Kurtz S, Shemesh G. Standard trabeculectomy and Ex-PRESS miniature glaucoma shunt: a comparative study and literature review. J Glaucoma. Epub 2014 March 13.

16. Netland PA, Sarkisian SR Jr, Moster MR, et al. Randomized, prospective, comparative trial of EX-PRESS glaucoma filtration device versus trabeculectomy (XVT study). Am J Ophthalmol. 2014;157:433-440.

17. Wagschal LD, Trope GE, Jinapriya D, Jin YP, Buys YM. Prospective randomized study comparing Ex-PRESS to trabeculectomy: 1-year results. J Glaucoma. Epub 2013 November 16.

18. Dahan E, Ben Simon GJ, Lafuma A. Comparison of trabeculectomy and EX-PRESS implantationin fellow eyes of the same patient: a prospective, randomized study. Eye (Lond). 2012;26:703-710.

19. Beltran-Agullo L, Trope GE, Jin Y, Wagschal LD, Jinapriya D, Buys YM. Comparison of visual recovery following Ex-PRESS versus trabeculectomy: results of a prospective randomized controlled trial. J Glaucoma. 2015;24:181-186
20. Kanner EM, Netland PA, Sarkisian SR Jr, Du H. Ex-PRESS miniature glaucoma device implanted under a scleral flap alone or combined with phacoemulsification cataract surgery. J Glaucoma. 2009;18:488-491.

21. Bagnis A, Papadia M, Scotto R, Traverso CE. Obstruction of the Ex-PRESS miniature glaucoma device: Nd: YAG laser as a therapeutic option. J Glaucoma. 2011;20:271.

22. Netland PA. Obstruction (also known as occlusion or blockage) of the Ex-PRESS miniature glaucoma device. J Glaucoma. 2011;20: 271-272.

23. Geffen N, Trope GE, Alasbali T, Salonen D, Crowley AP, Buys YM. Is the Ex-PRESS glaucoma shunt magnetic resonance imaging safe? J Glaucoma. 2010;19:116-118.

24. Seibold LK, Rorrer RA, Kahook MY. MRI of the Ex-PRESS stainless steel glaucoma drainage device. Br J Ophthalmol. 2011;95:251-254.

25. De Feo F, Roccatagliata L, Bonzano L, Castelletti L, Mancardi G, Traverso CE. Magnetic resonance imaging in patients implanted with Ex-PRESS stainless steel glaucoma drainage microdevice. Am J Ophthalmol. 2009;147:907-911.

26. Mabray MC, Uzelac A, Talbott JF, Lin SC, Gean AD. Ex-PRESS glaucoma filter: an MRI compatible metallic orbital foreign body imaged at 1.5 and 3T. Clin Radiol. 2015;70:e28-e34.

27. Sugiyama T, Shibata M, Kojima S, Ueki M, Ikeda T. The first report on intermediate-term outcome of EX-PRESS glaucoma filtration device implanted under scleral flap in Japanese patients. Clin Ophthalmol. 2011;5:1063-1066.

28. Patel HY, Wagschal LD, Trope GE, Buys YM. Economic analysis of the Ex-PRESS miniature glaucoma device versus trabeculectomy. J Glaucoma. 2014;23:385-390.
Medical Devices: Evidence and Research

\section{Publish your work in this journal}

Medical Devices: Evidence and Research is an international, peerreviewed, open access journal that focuses on the evidence, technology, research, and expert opinion supporting the use and application of medical devices in the diagnosis, treatment and management of clinical conditions and physiological processes. The identification of novel

\section{Dovepress}

devices and optimal use of existing devices which will lead to improved clinical outcomes and more effective patient management and safety is a key feature. The manuscript management system is completely online and includes a quick and fair peer-review system. Visit http://www. dovepress.com/testimonials.php to read real quotes from authors. 